\title{
LS-2
}

\section{Laparoscopic pancreatectomy for pancreatic cancer with advanced energy device (LigaSure Maryland jaw device)}

\author{
Chang Moo KANG*
}

Division of HBP Surgery, Department of Surgery, Yonsei University College of Medicine, Seoul, Korea

Lecture: Pancreatic cancer is well known to be one of the most lethal malignant diseases in gastrointestinal tract. As a monotherapy, margin-negative radical pancreatectomy is regarded as the most potent anticancer treatment. However, to enhance the oncologic efficacy of curative resection, postoperative chemotherapy should be considered. As a matter of fact, it is estimated that pancreatic cancer will be the second most common cancer-related human mortality in 2030, which will be main theme regarding human health issue. With advance of pancreatic surgery, developing method for early diagnosis, understanding tumor biology, and introduction of new potent chemotherapeutic agents, pancreatic cancer is expected to face new paradigm in near future.

Especially in treating pancreatic cancer, surgical extirpation is very important. Due to anatomical complexity, and surgical difficulty, laparoscopic radical pancreatectomy in treating pancreatic cancer is still controversial. However, currently available data are showing its feasibility, safety, and equivalent oncologic outcomes comparing with open surgical approach in well selected patients. There are potential theoretical advantages of laparoscopic pancreatectomy. Early recovery with less surgical stress is expected to provide the patients with appropriate patients' condition to receive the postoperative adjuvant chemotherapy, which is inevitable requirement for the long-term survival.

Therefore, in performing laparoscopic radical pancreatectomy for pancreatic cancer, surgeons need for effective energy device in dissecting the pancreas for safe and effective margin-negative resection. It is thought that LigaSure Maryland jaw device can be appropriate in performing laparoscopic radical pancreatectomy for pancreatic cancer due to following several reasons; 1) Burst pressure 2) Temperature 3) Nano-coating 4) Blunt dissection. Obtaining superior mesenteric artery (SMA) lateral margin is crucial in pancreatic head cancer. Very narrow space is allowed for securing SMA lateral margin. Alternative advanced energy device, Sonicision curved jaw cordless ultrasonic dissection system, can enhance the surgical performance and safety. Thin and small curved blade $(0.8 \mathrm{~mm}-1.7$ $\mathrm{mm}$ ) can provide easy access to the tight space with less potential thermal damage near the major vascular structure. In this presentation, I would like to discuss our clinical practice of laparoscopic pancreatectomy for pancreatic cancer with advanced energy device. 